\title{
Mitofusin2 regulates the proliferation and function of fibroblasts: The possible mechanisms underlying pelvic organ prolapse development
}

\author{
XIAOQING WANG ${ }^{1}$, XIAOXIAO WANG ${ }^{1}$, YINGFANG ZHOU $^{1}$, CHAO PENG $^{1}$, HUAYUN CHEN ${ }^{2}$ and YE LU ${ }^{1}$ \\ ${ }^{1}$ Department of Obstetrics and Gynecology, Peking University First Hospital, Beijing 100034; \\ ${ }^{2}$ Department of Obstetrics and Gynecology, Beijing Tsinghua Changgeng Hospital, Beijing 102218, P.R. China
}

Received August 23, 2018; Accepted June 6, 2019

DOI: $10.3892 / \mathrm{mmr} .2019 .10501$

\begin{abstract}
The present study aimed to investigate the effects of Mitofusin2 (Mfn2) on the proliferation of human uterosacral ligament fibroblasts and on the expression of procollagen. We also aimed to identify the possible signal transduction pathway involved in the development of pelvic organ prolapse (POP). For this purpose, uterosacral ligaments were harvested from POP and non-pelvic organ prolapse (NPOP) patients for fibroblast culture. Cellular proliferation and the cell cycle were assessed following transduction with lentiviral vectors for the overexpression and suppression of Mfn2. The expression levels of the proteins Mfn2, procollagens, phosphoprotein 21 wild-type $\mathrm{p} 53$ activating fragment (p21Waf1), cyclin-dependent kinase 2 (CDK2), extracellular signal-regulated kinase1/2 (ERK1/2) and rapidly accelerated fibrosarcoma-1 (Raf-1) were examined. Overexpression of Mfn2 resulted in the decreased proliferation of cells and the induction of G0/G1 phase arrest. Concomitantly, the relative expression levels of procollagen proteins, CDK2 and the phosphorylation levels of ERK1/2 and Raf-1 proteins were notably decreased, while the levels of the p21waf1 protein were increased in the Mfn2 overexpressing
\end{abstract}

Correspondence to: Professor Ye Lu, Department of Obstetrics and Gynecology, Peking University First Hospital, 1 Xi'anmen Street, Xicheng, Beijing 100034, P.R. China

E-mail: songqinlu@sina.com

Abbreviations: POP, pelvic organ prolapse; Mfn2, mitofusin2; NPOP, non-pelvic organ prolapse; p21Waf1, phosphoprotein 21 wild-type p53 activating fragment; CDK-2, cyclin-dependent kinase 2; ERK1/2, extracellular signal-regulated kinase1/2; Raf-1, rapidly accelerated fibrosarcoma-1; Mfn2+, NPOP fibroblasts with lentiviral vector of the open reading frame gene sequence of Mfn2; Mfn2-, NPOP fibroblasts transfected with lentiviral unloaded vector; sh, short hairpin RNA group, POP fibroblasts transfected with lentiviral vector containing shRNA interference sequence; CCK-8, cell counting kit- 8

Key words: pelvic organ prolapse, Mitofusin2, fibroblast, lentiviral vector, signaling pathway group. Opposing results were reported cells following Mfn2 silencing via RNA interference. The results of the present study indicated that the cell cycle of the fibroblasts, their cellular proliferation and the levels of the procollagen proteins could be inhibited via the Ras-Raf-ERK axis as a result of the increased levels of Mfn2 during the development of POP.

\section{Introduction}

The incidence of pelvic organ prolapse (POP) has increased during the last few years (1). This condition notably affects the quality of life of many females worldwide; however, at present, several studies have reported the lack of effective preventative methods for patients with high risk factors of POP (2), and deficiencies in strategies to suppress the development of POP (1-8). Previous studies have notably focused on the clinical diagnosis and treatment of POP, and the mechanism of action associated the extracellular matrix (ECM) (9-13). Few studies have investigated the proliferation and activity of fibroblasts, which are associated with the synthesis and secretion of procollagen (14).

Mfn2 serves an important role in signal transduction, the prevention of sustained extensive mitochondrial elongation, the activation of cellular senescence and the induction of apoptosis (15-18). It is important to note that Mfn2 controls cell metabolism by limiting the production of reactive oxygen species and by modulating the endoplasmic reticulum stress (15). Thus, the mitochondrial fusion protein mitofusin 2 (Mfn2) is a cellular hub that senses the metabolic and hormonal milieu, and drives the control of metabolic homeostasis (15). Abnormal expression of Mfn2 can cause adverse effects. Previous studies have reported that Mfn2 is associated with lung diseases $(18,19)$ and the incidence of cancer (19). A limited number of studies have focused on pelvic floor diseases. POP is a common disease in the elderly population, which is associated with the damage and aging of the pelvic floor support structure $(1,20)$. Furthermore, Mfn2 is associated with aging (17). Our previous study (21) demonstrated a correlation between Mfn2 expression levels and POP in vivo. Fibroblasts were isolated from uterosacral ligaments using laser capture microdissection. The results indicated that the expression levels of Mfn2 in patients with POP increased 
and the expression levels of the procollagen protein decreased compared with those of non-pelvic organ prolapse (NPOP) samples (21). The successive study of our group (22) demonstrated in vitro that the proliferation of primary cultured fibroblasts and the expression levels of procollagen protein had decreased, while the expression levels of Mfn2 were increased in POP uterosacral ligaments compared with NPOP samples. Following the inhibition of Mfn2 expression, the levels of procollagen protein were increased. Based on these previous findings, we further examined alterations in the cellular proliferation of fibroblasts, their progression through the various stages of the cell cycle and the expression levels of procollagen proteins following upregulation of Mfn2. The present study aimed to determine the possible mechanism underlying the pathogenesis of POP.

\section{Materials and methods}

Study subjects. Uterosacral ligaments were collected from 10 POP and 10 NPOP cases during hysterectomy in the Peking University First Hospital from September 2016 to December 2016. After hysterectomy, fresh uterosacral ligament tissues were cut into pieces with a diameter $<0.1 \mathrm{~cm}$, these tissues pieces were cultured in Dulbecco's Modified Eagle's medium Nutrient Mixture F-12 (Ham) (DMEM/F12; Gibco; Thermo Fisher Scientific) containing 20\% FBS (Gibco; Thermo Fisher Scientific) and $1 \%$ antibiotic solution in an incubator at $37^{\circ} \mathrm{C}$ containing $5 \% \mathrm{CO}_{2}$. Primary culture was conducted until 2-4 colonies were formed, which were used for transfection. The POP group comprised stage III to IV cases (according to the POP-Q score) (23). The NPOP group was matched with patients who required hysterectomy due to benign gynecological diseases, including hysteromyoma and adenomyosis, according to age (years; POP, $55.60 \pm 11.33$ and NPOP, $50.70 \pm 7.90$ ), body mass index (POP, $23.57 \pm 3.33$ and NPOP, $23.03 \pm 2.91$ ), parity (POP, $1.40 \pm 0.70$ and NPOP, $1.20 \pm 0.42$ ) and postmenopausal duration (years; POP, 5.75 \pm 9.59 and NPOP, $1.90 \pm 6.01)(n=10, P>0.05$, all). All patients exhibited no history of urinary tract infection, estrogen drugs, vaginal surgery and/or diseases that could affect the metabolism of collagen during the 3 month period prior to the study. The clinical data of patients is presented in Table I. Samples were collected after informed consent from patients was obtained. The present study was approved by the ethics committee of Peking University First Hospital [approval no. 2016 (1173)].

Main reagents and instruments. Anti-extracellular-signal regulated kinase 1/2 (ERK1/2; diluted 1/200, sc-135900), anti-phosphorylated (p)ERK1/2 (diluted 1/200, sc-377400), anti-Raf-1 (diluted 1/200, sc-52827), anti-pRaf-1 (diluted 1/200, sc-293351), anti-p21waf1 (diluted 1/200, sc-90110), anti-cyclin dependent kinase 2 (CDK2; diluted 1/200, sc-136191), anti-procollagen 1A1/1A2/3A1 (diluted 1/200, sc-133179, sc-166572, sc-16333, respectively) were purchased from Santa Cruz Biotechnology, Inc. Anti- $\beta$-tubulin monoclonal antibody (diluted $1 / 1,000$, TA347064) and anti- $\beta$-actin monoclonal antibody (diluted 1/1,000, TA-09) were obtained from ZSGB-BIO. Anti-Mfn2 monoclonal antibody (diluted 1/500, ab56889) was obtained from Abcam. The cell cycle detection kit, myllicin mixture (KGY0023), Pancreatin (KGY001) and
Pancreatin-EDTA (KGY0012) were obtained from Nanjing KeyGen Biotech Co., Ltd. The cell counting kit-8 (CCK-8) was purchased from Dojindo Molecular Technologies, Inc. DMSO was obtained from AppliChem $\mathrm{GmbH}$; Enhanced Chemiluminescent Reagent was purchased from GE Healthcare Life Sciences. Fetal bovine serum (cat. no. 10099-141) was purchased from Gibco (Thermo Fisher Scientific, Inc.). The mouse anti-human cytokeratin monoclonal (CK-19), horseradish peroxidase-labeled goat anti-mouse IgG (ZB-2305) antibodies and peroxidase-conjugated goat anti-rabbit $\operatorname{IgG}$ (ZB-2301) were purchased from OriGene Technologies, Inc.

Lentiviral vectors. Mfn2-overexpressing and RNA interference (RNAi) lentiviral vectors were provided by Shanghai GeneChem Co., Ltd. The lentiviral vectors employed for Mfn2 overexpression and downregulation were Ubi-MCS-3FLAG-SV40-enhanced green fluorescent protein (EGFP) and U6-MCS-Ubi-EGFP, respectively. The RNAi sequences for Mfn2 and the negative control sample were ACTTTGTCACTGCCAAGAA and GTTCTCCGAACGTGT CACGT, respectively.

Primary cell culture, subculture, cryopreservation and resuscitation. Uterosacral ligament tissues were harvested during hysterectomy. The tissue fragments possessed dimensions of $0.5 \times 0.5 \times 0.5 \mathrm{~cm}^{3}$. The fibroblasts could be observed under a light microscope (magnification, $\mathrm{x} 4$ ) after 1-2 weeks of primary culture. The cells were collected for subculture (passage 2-4) and/or long term storage at $-80^{\circ} \mathrm{C}$ when the confluence of the cell culture was $\sim 70-80 \%$. The procedure was conducted in a biosafety cabinet. For resuscitation, frozen cells were removed from $-80^{\circ} \mathrm{C}$ and warmed rapidly in water at $37^{\circ} \mathrm{C}$. The contents of the tube was added to $10 \mathrm{X}$ volume of culture medium and centrifuged at $300 \mathrm{x}$ g for $5 \mathrm{~min}$. The cell pellet was resuspended in medium (containing 20\% FBS and $1 \%$ antibiotics) and placed in an incubator at $37^{\circ} \mathrm{C}$.

Cell groups. Fibroblasts in the NPOP group were used for the Mfn2-overexpression experiments. The cells were divided into three groups as follows: Mfn2+ group, comprising NPOP fibroblasts transduced with lentiviral vector containing the open reading frame of Mfn2; Mfn2- group, NPOP fibroblasts were transduced with an empty lentiviral vector and the control group, comprising non-transduced NPOP fibroblasts. Concomitantly, POP fibroblasts were used for Mfn2 knockdown experiments according to the following three groups: Short hairpin RNA group (sh-Mfn2 group), comprising POP fibroblasts transduced with lentiviral vector containing the shRNA interference sequence; short hairpin RNA negative control (sh-Mfn2-NC) group that included POP fibroblasts transduced with lentiviral vector containing a shRNA negative control (NC) sequence and the control group, comprising non-transduced POP fibroblasts.

Transduction. Prior to infection, the cells in the logarithmic growth phase that were grown in the presence of antibiotic-free medium were prepared into a cell suspension at a concentration of $2-3 \times 10^{4}$ cells $/ \mathrm{ml}$ with pancreatin-EDTA. The cell suspension was added to each well and cultured at $37^{\circ} \mathrm{C}$, in the presence of $5 \% \mathrm{CO}_{2}$. Adherent cells that 
Table I. Comparison of clinical data between POP and NPOP patients.

\begin{tabular}{|c|c|c|c|c|}
\hline Parameters (mean \pm standard error of the mean) & $\operatorname{NPOP}(n=10)$ & $\operatorname{POP}(n=10)$ & t-value & P-value \\
\hline Age (year) & $50.70 \pm 7.90$ & $55.60 \pm 11.33$ & 1.122 & 0.277 \\
\hline BMI $\left(\mathrm{kg} / \mathrm{m}^{2}\right)$ & $23.03 \pm 2.91$ & $23.57 \pm 3.33$ & 0.348 & 0.732 \\
\hline Age of first delivery (years) & $27.14 \pm 3.80$ & $24.75 \pm 3.45$ & 1.277 & 0.224 \\
\hline Parity (n) & $1.20 \pm 0.42$ & $1.40 \pm 0.70$ & 0.775 & 0.449 \\
\hline Postmenopausal duration (years) & $1.90 \pm 6.01$ & $5.75 \pm 9.59$ & 1.043 & 0.312 \\
\hline
\end{tabular}

Data were acquired via an independent samples t-test. BMI, body mass index; POP, pelvic organ prolapse, NPOP, non-pelvic organ prolapse. No significant differences were observed between the two groups.

attained $20-30 \%$ confluence were observed under an inverted microscope at a magnification of $\mathrm{x} 4$ (5 fields per view) the following day. The virus was incubated with the cells in $10 \% \mathrm{FBS}+\mathrm{DMEM} / \mathrm{F} 12$ in the absence of antibiotics. The ratio of lentiviral particles to the number of cells (multiplicity of infection) was 20:1. The final concentration of the viral particles was $5 \mathrm{~g} / \mathrm{ml}$ and Polybrene was employed for transduction. During viral transfection, the cells were washed two times with PBS and 10-12 h later, the complete culture medium was used to replace the medium containing the virus. The expression of GFP was observed under fluorescence microscope (magnification, $x 40 ; 5$ fields per view) following $96 \mathrm{~h}$ of incubation.

Detection of fibroblast proliferation by a CCK- 8 assay. A CCK-8 assay was conducted to detect cell proliferation in each group $96 \mathrm{~h}$ following transduction with the lentivirus. The steps were as follows: The cell suspension was diluted to the specific cell densities, including $8 \times 10^{4}, 4 \times 10^{4}, 2 \times 10^{4}$ and $1 \times 10^{4}$ cells $/ \mathrm{ml}$, and the cells were incubated in a 96 -well plate at a volume of $100 \mu \mathrm{l} /$ well at $37^{\circ} \mathrm{C}$. Medium was added to the edge of wells. A total of $100 \mu \mathrm{l}$ of CCK-8 containing medium (10\% FBS: CCK-8=9:1) was added $24 \mathrm{~h}$ later. Following $2 \mathrm{~h}$ of incubation at $37^{\circ} \mathrm{C}$, the absorbance was measured with a microplate reader at the wavelength of $450 \mathrm{~nm}$ (OD). Similarly, a standard curve was generated using the number of cells as the abscissa ( $\mathrm{X}$ axis) and the OD value for the vertical axis ( $\mathrm{Y}$ axis). A new 96-well plate was used every day and measurements were recorded on days $1,3,5,7,9$ and 11 . The cell proliferation curves were plotted according to the data obtained from these six time points.

Cell cycle analysis. The cells were collected using $0.25 \%$ no-EDTA trypsin for $1-2 \mathrm{~min}$ at $37^{\circ} \mathrm{C}$ following $96 \mathrm{~h}$ of viral infection and subsequently washed with PBS. The samples were centrifuged at $300 \mathrm{x} \mathrm{g}$ at room temperature for $5 \mathrm{~min}$ and the supernatant was discarded. The cells were fixed at $4^{\circ} \mathrm{C}$ for $4 \mathrm{~h}$ using $75 \%$ ethanol, centrifuged at $500 \mathrm{x}$ g for $5 \mathrm{~min}$ at room temperature and incubated with $100 \mu \mathrm{l}$ RNase A $(100 \mu \mathrm{g} / \mathrm{ml})$ in a $37^{\circ} \mathrm{C}$ water bath for $30 \mathrm{~min}$ in the presence of ethidium bromide (Sigma-Aldrich; Merck KGaA). Flow cytometry (FCM) was performed within $1 \mathrm{~h}$ with a flow cytometer. Each group analysis was repeated three times and the mean value was obtained. The results were analyzed with ModFit LT 4.1 software (Verity Software House, Inc.).
Protein expression assays (Mfn2, procollagen, pERK1/2, pRaf-1, p21waf1, CDK2). The relative expression of proteins was obtained by western blotting following protein extraction using RIPA Lysis Buffer [RIPA (Beyotime Institute of Biotechnology): PMSF (Beyotime Institute of Biotechnology): Phosphatase Inhibitor cocktail I (MedChemExpress LLC): Phosphatase Inhibitor cocktail II (MedChemExpress LLC) at a ratio of 100:1:1:1] from $10^{6}$ cells. The bicinchoninic acid method was used to determine protein concentration. The proteins (15 $\mu \mathrm{g} /$ lane) were separated by electrophoresis using a $10 \%$ SDS-PAGE gel and transferred to a PVDF membrane. The membrane was blocked with 5\% milk in TBS-Tween-20 and incubated with primary antibodies for the proteins Mfn2, procollagen 1A1/1A2/3A1, pRaf-1, pERK1/2, p21waf1, CDK2 and $\beta$-actin overnight at $4^{\circ} \mathrm{C}$. The following morning, the membrane was washed three times with TBS-T and incubated with a secondary goat anti mouse IgG antibody conjugated with horseradish peroxidase (diluted 1:5,000) at room temperature for $1 \mathrm{~h}$. Membranes were washed three times and ECL was used for visualization. Experiments were repeated three times. Image $\mathrm{J}$ for Mac (1.47d; National Institutes of Health) was used to scan the specific bands for each protein and the ratio of the target protein bands to the $\beta$-actin bands was calculated.

Statistical analysis. Experiments were repeated three times and the results were expressed as the mean \pm standard deviation. SPSS 23 (IBM, Corp.) was used for analysis. Independent samples t-test and general linear model-univariate analysis and/or one way ANOVA were used for comparison between different groups when the data followed normal distributions. Bonferroni correction was used when the variance was equal and Dunnett's T3 post hoc test was used if the variance was not equal. For non-normal distributions, between group comparisons were assessed using Wilcoxon and/or Kruskal-Wallis tests, and the rank sum statistic $\chi^{2}$ was used. $\mathrm{P}<0.05$ was considered to indicate a statistically significant difference.

\section{Results}

Inhibitory effects of Mfn2 on fibroblast proliferation. The primary fibroblasts observed via light microscopy were presented in Fig. 1. The data verified the identification of the fibroblasts as reported in our previous study (22). Following 11 days of the initial cell seeding, the cell activity was significantly reduced in the Mfn2-overexpressing model compared 

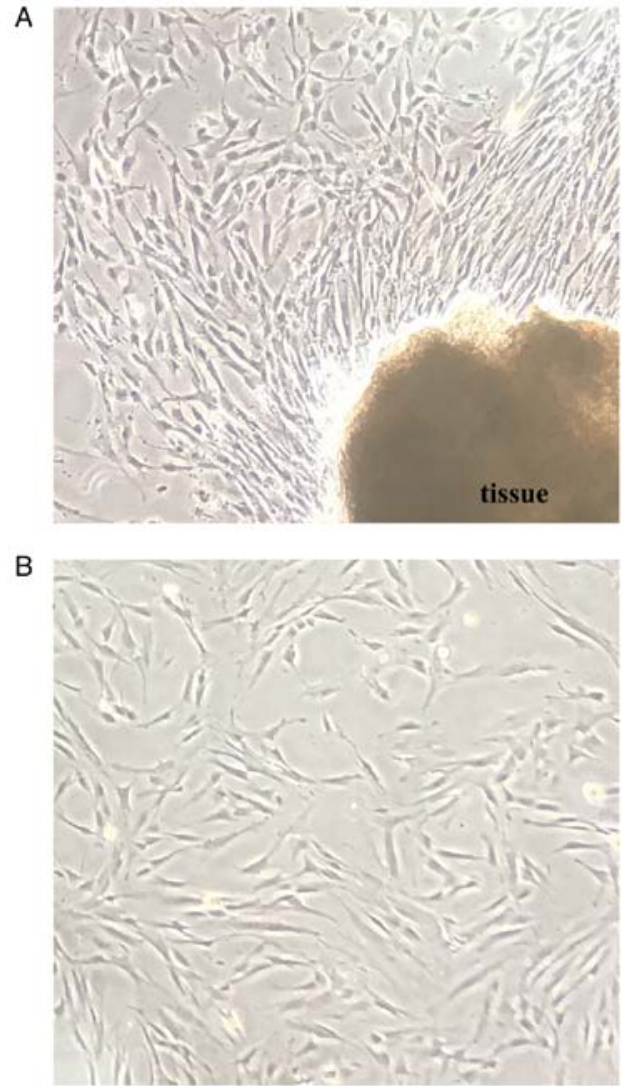

Figure 1. Fibroblasts observed under a light microscope. (A) Fibroblasts observed under a light microscope (magnification, $\mathrm{x} 4$ ) following 1 week or of primary culture. (B) The cells were collected for subculture.

with that of the control and the Mfn2- groups. The absorbance of the Mfn2+, Mfn2- and control groups, respectively on day 1 were determined to be $0.138 \pm 0.003,0.147 \pm 0.002$ and $0.144 \pm 0.003$, respectively. Similarly, the corresponding cell proliferative activities were analyzed for the remaining time periods and were as follows: Day $3,0.206 \pm 0.003 ; 0.211 \pm 0.003$ and $0.210 \pm 0.004$; day $5,0.251 \pm 0.003 ; 0.372 \pm 0.004$ and $0.425 \pm 0.004$; day $7,0.347 \pm 0.004 ; 0.767 \pm 0.003$ and $0.832 \pm 0.004$; day $9,0.513 \pm 0.004 ; 1.406 \pm 0.003$ and $1.353 \pm 0.005$ and day 11 $0.606 \pm 0.004 ; 1.696 \pm 0.005$ and $1.781 \pm 0.005$ for the respective Mfn2+, Mfn2- and control groups. No significant differences were noted between the two control groups $(\mathrm{P}>0.05)$. From days 5-11, the proliferation of Mfn2+ fibroblasts was significantly reduced compared with the control groups (Fig. 2; $\mathrm{P}<0.001)$.

The activity of the sh-Mfn2 group fibroblasts was significantly increased in the sh-Mfn2 cells compared with that of the sh-Mfn2-NC and control groups $(\mathrm{P}<0.001$, from days 5-11). The absorbance of the sh-Mfn2, sh-Mfn2-NC and control groups, respectively at the different time periods were as follows: Day 1, 0.142 $\pm 0.003,0.144 \pm 0.003$ and $0.142 \pm 0.002$; day $3,0.206 \pm 0.004,0.212 \pm 0.004$ and $0.210 \pm 0.004$; day $5,0.401 \pm 0.004,0.305 \pm 0.021$ and $0.234 \pm 0.004$; day $7,0.729 \pm 0.004,0.338 \pm 0.004$ and $0.331 \pm 0.004$; day 9 , $1.067 \pm 0.004,0.508 \pm 0.003$ and $0.418 \pm 0.004$, and day 11 , $1.483 \pm 0.005,0.596 \pm 0.004$ and $0.530 \pm 0.004$ for the respective sh-Mfn2, sh-Mfn-NC and control groups. The proliferation of sh-Mfn2 fibroblast significantly increased from days 5-11
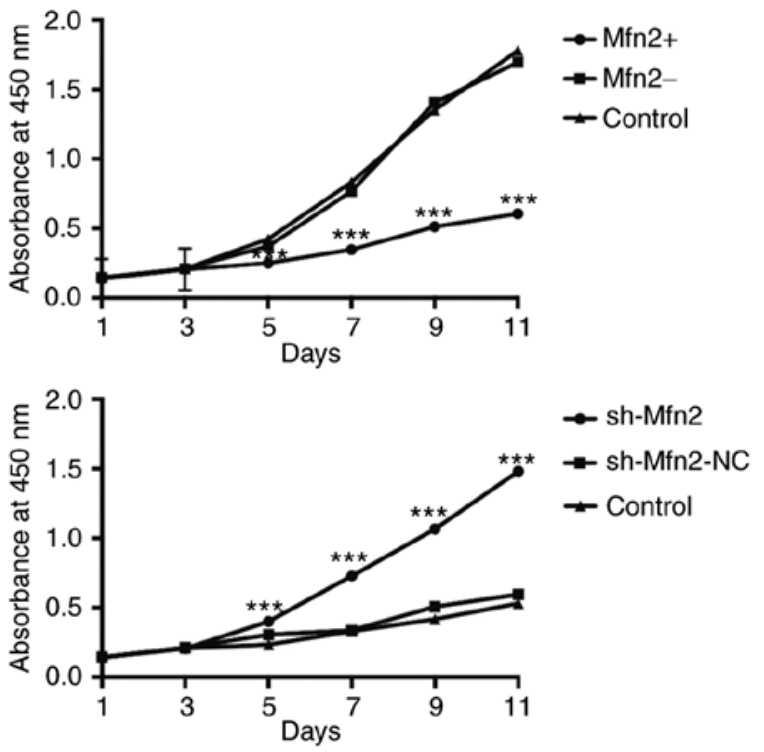

Figure 2. Fibroblast proliferation as examined by a Cell Counting Kit-8 assay. In Mfn2-overexpressing cells, cell activity was significantly reduced compared with the control and Mfn2- groups $\left(\mathrm{n}=10,{ }^{* * * *} \mathrm{P}<0.001\right.$ vs. control and Mfn2-). In the Mfn2-inhibition group, the activity of sh-Mfn2 fibroblasts was significantly increased compared with that of the sh-Mfn2-NC and the control groups $\left(\mathrm{n}=10,{ }^{* * *} \mathrm{P}<0.001\right.$ vs. control and sh-Mfn2-NC). A t-test and repeated measurement data analysis of variance were used to determine statistical significance compared with the control groups. The experiments were repeated for three times, indicating consistent results. GFP, green fluorescent protein; Mfn2, mitofusin 2; NC, negative control; NPOP, non-pelvic organ prolapse; sh, short hairpin RNA; control, non-transduced NPOP cells (top panel), non-transfected POP cells (bottom panel); Mfn2+, NPOP cells infected with LV-Mfn2-GFP; Mfn2-, NPOP cells transfected with LV-GFP; sh-Mfn2, POP cells transfected with LV-sh-Mfn2-GFP; sh-Mfn2-NC, POP cells transfected with LV-sh-Mfn2-NC-GFP.

$(\mathrm{P}<0.001)$; however, no significant difference was observed between the two control groups $(\mathrm{P}>0.05)$ (Fig. 2).

Inhibitory effects of Mfn2 on fibroblast cell cycle. The ratio of the percentage of fibroblasts at the G0/G1 phase in the Mfn2+ group $(74.939 \pm 0.562 \%)$ was markedly increased compared with that of the Mfn2- $(36.526 \pm 0.549 \%)$ and the control groups $(44.159 \pm 0.461 \%$; $n=10)$. Compared with the population of the cells in the G1 phase, the proportion of cells in the $\mathrm{S}$ and $\mathrm{G} 2 / \mathrm{M}$ phases in the Mfn2+ group was markedly lower compared with that of the control group. No differences were noted between the two control groups (Fig. 3A).

In the Mfn2 knockdown group, the number of cells in the G0/G1 phase was markedly lower $(41.469 \pm 0.797 \%)$ compared with the sh-Mfn2-NC (61.606 $\pm 0.711 \%)$ and the control groups $(57.649 \pm 0.795 \%)$. By contrast, the proportion of the cells in $\mathrm{S}$ and G2/M phases of the sh-Mfn2 group was markedly higher than that of the control group. The cell percentages of the two control groups indicated no difference (Fig. 3).

Relative expression of Mfn2 and procollagen proteins. The relative expression of each protein was detected by western blot analysis. The expression of the proteins following the knockdown of Mfn2 in POP fibroblasts has been demonstrated in our previous study (21). In NPOP fibroblasts infected with lentivirus for the overexpression of Mfn2, the

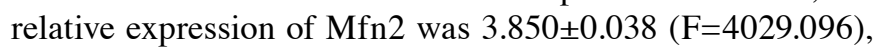



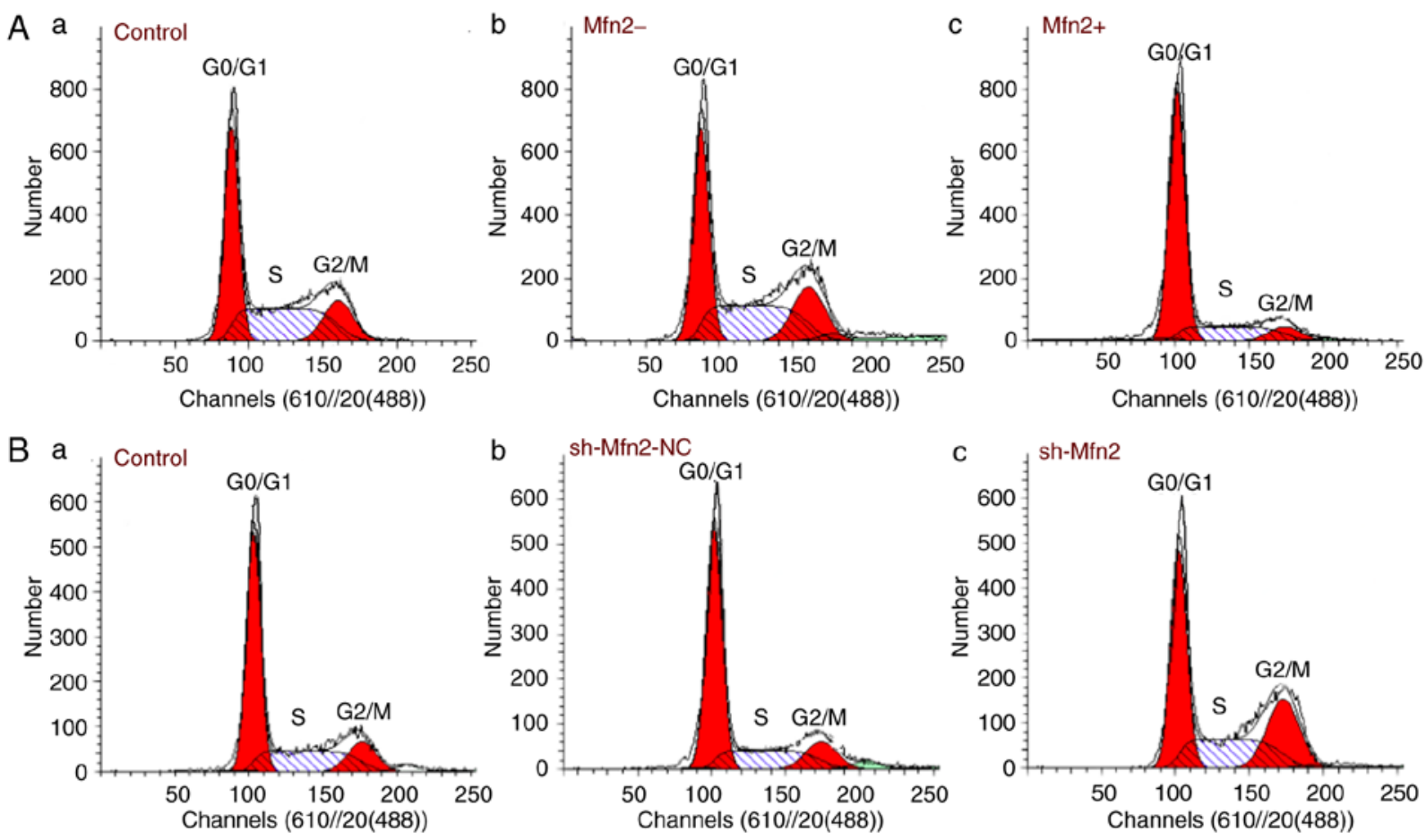

Figure 3. Cell cycle distribution following lentiviral transduction as detected by flow cytometry. (A) Significant differences in S and G2/M phases were noted between the Mfn2+ and the control groups. $\mathrm{n}=10(\mathrm{P}<0.05)$. (B) The proportion of cells in the $\mathrm{S}$ and $\mathrm{G} 2 / \mathrm{M}$ phases of the sh-Mfn2 group was significantly higher than the control groups $n=10$. $(\mathrm{P}<0.05)$. A t-test was used to determine statistical significance. The experiments were repeated three times. Data are presented as the mean \pm standard error of the mean. GFP, green fluorescent protein; Mfn2, mitofusin 2; NPOP, non-pelvic organ prolapse; sh, short hairpin RNA; control, non-transduced NPOP cells (top panel, A-a), non-transduced POP cells (bottom panel, B-a); Mfn2+, NPOP cells infected with LV-Mfn2-GFP (A-c); Mfn2-, NPOP cells transduced with LV-GFP(A-b); sh-Mfn2, POP cells transduced with LV-sh-Mfn2-GFP(B-c); sh-Mfn2-NC, POP cells transduced with LV-sh-Mfn2- negative control - GFP (B-b).

which was significantly increased compared with the control $(1.081 \pm 0.016)$ and Mfn2- $(1.056 \pm 0.014)$ groups $(\mathrm{P}<0.001)$. No significant difference was noted between the two control groups $(\mathrm{P}>0.05)$. In addition, the relative expression levels of procollagen $1 \mathrm{~A} 1 / 1 \mathrm{~A} 2 / 3 \mathrm{~A} 1$ in the Mfn2+ group $(0.384 \pm 0.005$, $0.303 \pm 0.010,0.486 \pm 0.009$, respectively) were significantly decreased compared with those in the Mfn2- $(1.676 \pm 0.034$, $0.652 \pm 0.009,1.003 \pm 0.014$, respectively) and the control groups $(1.755 \pm 0.048,0.630 \pm 0.015,1.059 \pm 0.023$, respectively) $(\mathrm{P}<0.001)$. No significant difference was noted between the two control groups $(\mathrm{P}>0.05)$ (Fig. 4).

Phosphorylation of ERK1/2 and Raf-1 proteins following $M f n 2$ overexpression. Following $96 \mathrm{~h}$ of lentiviral transfection, total cellular protein was extracted from fibroblasts. The expression levels of total and phosphorylated levels of ERK1/2 and Raf-1 proteins were detected by western blot analysis (Fig. 5). The levels of total ERK1/2 protein expression were notably similar in the Mfn2+, Mfn2- and control groups $(1656.461 \pm 88.422,1642.728 \pm 61.549,1674.064 \pm 44.134$, respectively). However, the levels of pERK $1 / 2$ proteins in the Mfn2+ group $(0.120 \pm 0.014)$ were markedly decreased compared with the Mfn2- $(0.38 \pm 0.027)$ and control groups $(0.353 \pm 0.021)$. The expression profile of total Raf-1 expression was similar to that of ERK.

Following the inhibition of Mfn2 expression in POP fibroblasts, the expression levels of total ERK1/2 protein in the sh-Mfn2, sh-Mfn2-NC and control groups markedly varied $(1491.477 \pm 37.892,1491.639 \pm 49.668,1482.802 \pm 34.900$, respectively, $\mathrm{P}>0.05)$. The levels of $\mathrm{pERK} 1 / 2$ in the sh-Mfn2 group $(0.805 \pm 0.027)$ were significantly increased compared with the sh-Mfn2-NC $(0.3305 \pm 0.001)$ and the control groups $(0.5123 \pm 0.036)(\mathrm{P}<0.05)$. The trend in expression of total Raf and pRaf was similar to that of ERK1/2 and pERK1/2, respectively (Fig. 5B).

Inhibitory effects of Mfn2 on the expression of p21wafl and $C D K-2$. Following lentiviral transfection for $96 \mathrm{~h}$, total protein was extracted from fibroblasts. The relative expression levels of p21waf1 and CDK2 were semi-quantitatively analyzed by western blotting (Figs. 6 and 7).

In NPOP fibroblasts, the expression levels of p21waf1 in the Mfn2+ group $(0.322 \pm 0.026)$ were significantly upregulated than in the Mfn2- $(0.110 \pm 0.001)$ and control groups $(0.107 \pm 0.001)(\mathrm{P}<0.001)$. No significant difference was noted between the two control groups. In addition, the relative expression levels of CDK2 in the Mfn2+ group $(0.108 \pm 0.014)$ were significantly decreased compared with the Mfn2$(0.166 \pm 0.005)$ and control groups $(0.168 \pm 0.006)\left(\chi^{2}=15.768\right.$, $\mathrm{P}<0.01)$. No significant difference was noted between the two control groups ( $\mathrm{P}>0.05)$ (Fig. 6).

The expression levels of the p21waf1 protein in the sh-Mfn2 group $(0.170 \pm 0.015)$ were significantly downregulated than in the sh-Mfn2-NC $(0.328 \pm 0.003)$ and control groups $(0.333 \pm 0.004)$ following transfection of RNAi lentiviral particles in POP fibroblasts $\left(\chi^{2}=59.874, \mathrm{P}<0.001\right)$ (Fig. 7). No significant difference was reported between the sh-Mfn2-NC and control groups $(\mathrm{P}>0.05)$. In addition, the 

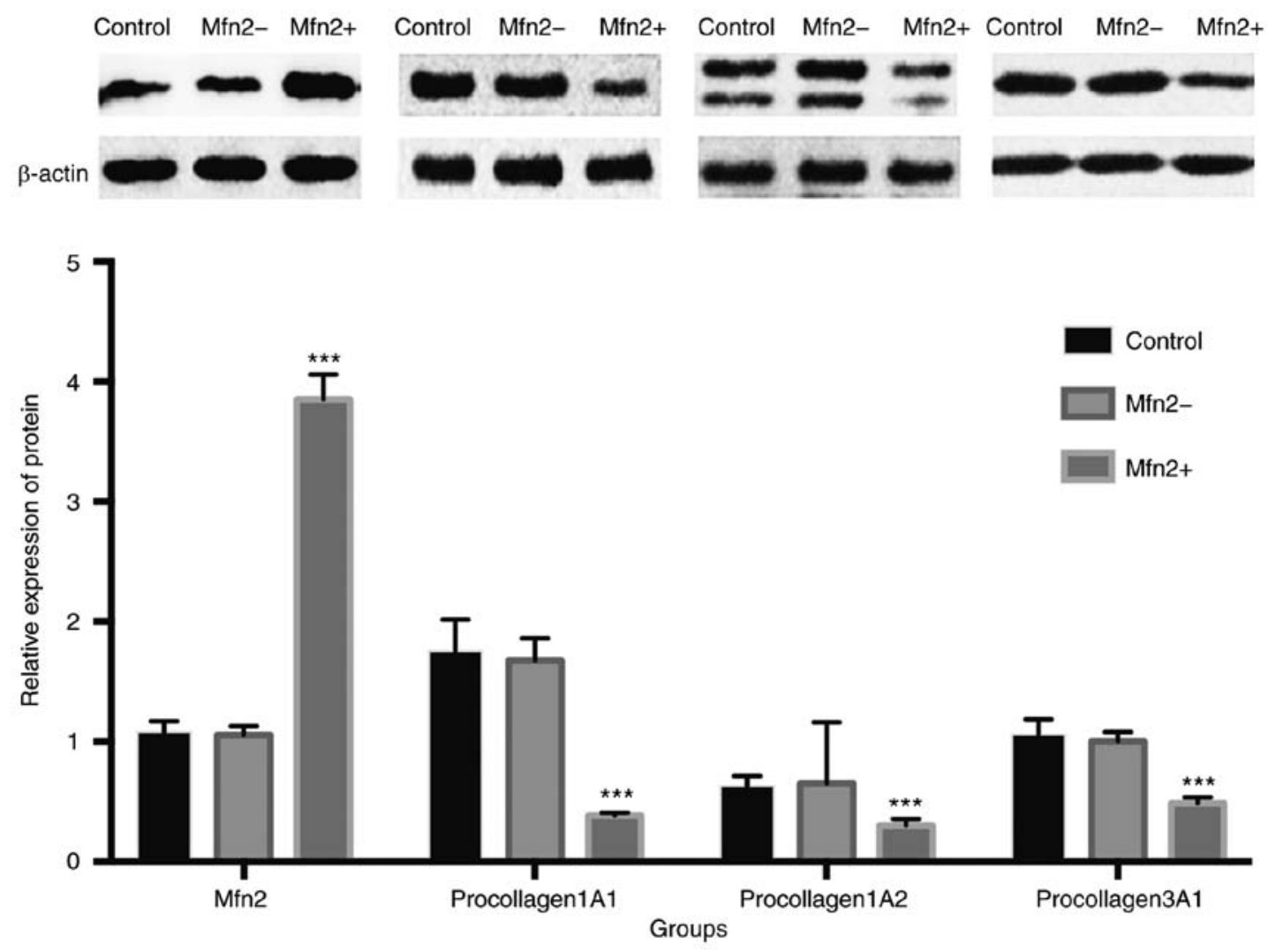

Figure 4. Expression levels of proteins in NPOP fibroblasts. In the Mfn2+ NPOP group, the relative expression levels of procollagen proteins in the were significantly downregulated than in the two control groups. ${ }^{* * *} \mathrm{P}<0.001 \mathrm{vs}$. control and Mfn2- groups. GFP, green fluorescent protein; Mfn2, mitofusin 2; NPOP, non-pelvic organ prolapse; control, non-transduced NPOP cells; Mfn2+, NPOP cells infected with LV-Mfn2-GFP; Mfn2-, NPOP cells transduced with LV-GFP.
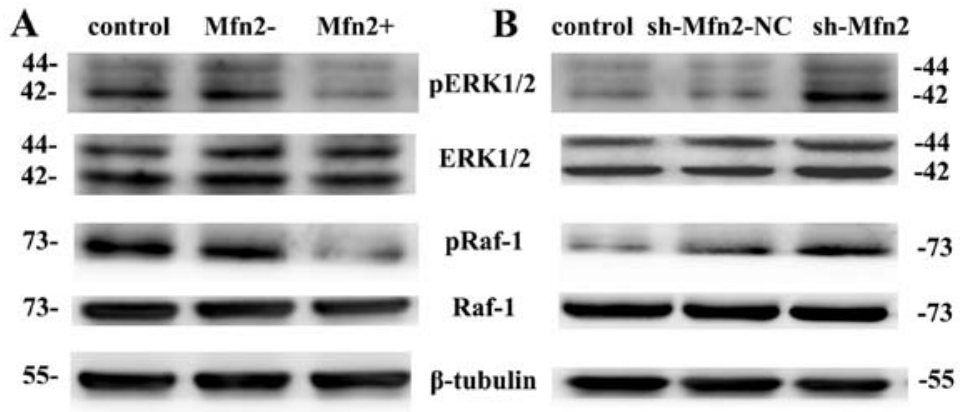

Figure 5. Expression levels of pERK1/2 and pRaf-1 proteins following lentiviral transfection of fibroblasts. (A) The expression levels of pERK1/2 and pRaf-1 proteins in the Mfn2+ group were significantly lower than in the Mfn2- and control groups. (B) The phosphorylation levels of ERK1/2 and Raf-1 proteins in the sh-Mfn2 group were markedly higher than in the sh-Mfn2-NC and control groups. ERK1/2, extracellular signal-regulated kinase1/2; GFP, green fluorescent protein; Mfn2, mitofusin 2; NC, negative control; NPOP, non-pelvic organ prolapse; p, phosphorylated; sh, short hairpin RNA; control, non-transduced NPOP cells, non-transduced POP cells; Mfn2+, NPOP cells infected with LV-Mfn2-GFP; Mfn2-, NPOP cells transduced with LV-GFP; sh-Mfn2, POP cells transduced with LV-sh-Mfn2-GFP; sh-Mfn2-NC, POP cells transduced with LV-sh-Mfn2-NC-GFP.

relative expression levels of CDK2 in the sh-Mfn2 group $(0.228 \pm 0.016)$ were significantly upregulated compared with the sh-Mfn2-NC $(0.084 \pm 0.001)$ and control groups $(0.082 \pm 0.002)\left(\chi^{2}=49.541, \mathrm{P}<0.001\right)$. No significant difference was noted between the two control groups $(\mathrm{P}>0.05)$.

\section{Discussion}

To examine the effects of Mfn2 on fibroblasts, we transduced fibroblasts with lentiviral vectors for the overexpression and knockdown of Mfn2. These vectors are suitable for achieving stable expression of sh-Mfn2 RNA and can significantly improve the transduction efficiency of specific cells that are difficult to transfect, such as primary cells and stem cells (24). In the present study, the infection efficiencies of Mfn2 overexpressing and RNAi lentiviral vectors were $>70 \%$, indicating that they could be used successfully for transfection.

The CCK- 8 assay is a colorimetric assay used to measure cell proliferation. The formation of the color is directly 

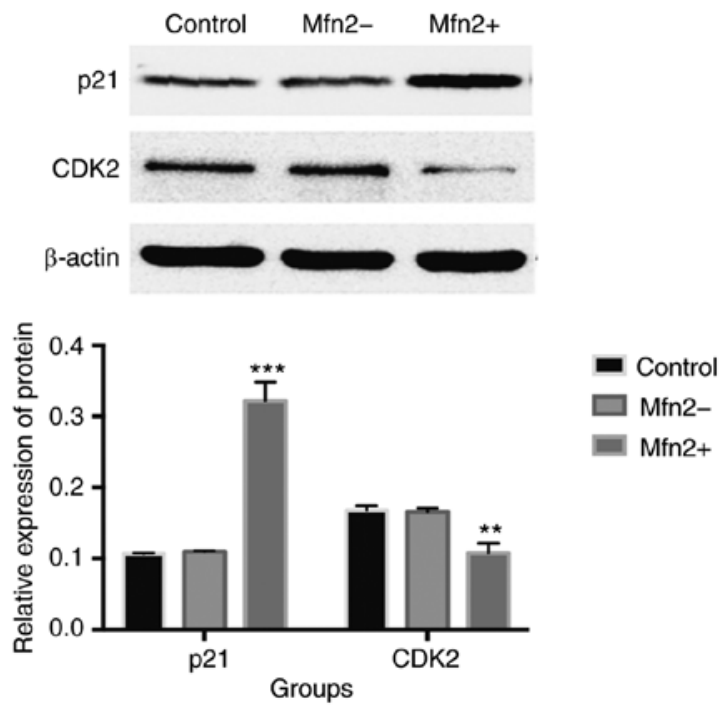

Figure 6. Expression levels of p21 and CDK2 proteins following lentiviral transfection of NPOP fibroblasts. The relative expression levels of CDK2 in the Mfn2+ group were significantly downregulated, while p21 was upregulated compared with the Mfn2- and control groups. ${ }^{* *} \mathrm{P}<0.01$ and ${ }^{* * *} \mathrm{P}<0.001$ vs. control and Mfn2- groups. CDK2, cyclin-dependent kinase 2; GFP, green fluorescent protein; Mfn2, mitofusin 2; NPOP, non-pelvic organ prolapse; control, non-transduced NPOP cells; Mfn2+, NPOP cells infected with LV-Mfn2-GFP; Mfn2-, NPOP cells transduced with LV-GFP.

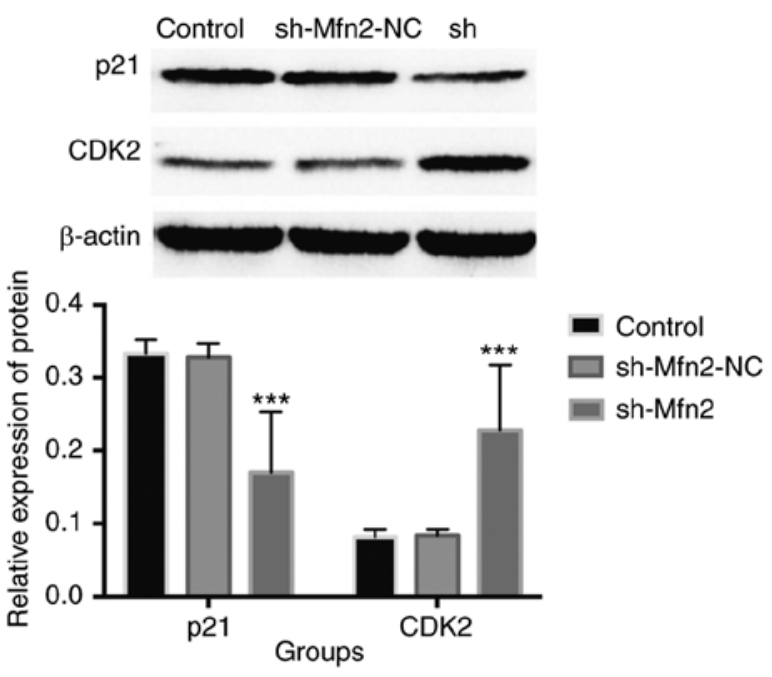

Figure 7. Expression levels of p21 and CDK2 proteins following lentiviral transduction of POP fibroblasts. The relative expression levels of CDK2 in the sh-Mfn2 group were significantly decreased, while CDK2 was upregulated compared with the sh-Mfn2-NC and control groups. ${ }^{* * *} \mathrm{P}<0.001$ vs. the control and sh-Mfn2-NC groups. CDK2, cyclin-dependent kinase 2; GFP, green fluorescent protein; Mfn2, mitofusin 2; NC, negative control; POP, pelvic organ prolapse; control, non-transduced POP cells; sh-Mfn2, POP cells transduced with LV-sh-Mfn2-GFP; sh-Mfn2-NC, POP cells transduced with LV-sh-Mfn2-NC-GFP.

proportional to the proliferation of cells. The use of a microplate reader to determine the OD indirectly reflects the number of living cells. The present study indicated that the cell activity in the Mfn2+ group was significantly reduced compared with that noted in the two control groups, whereas in the sh-Mfn2 group, the activity of the sh-Mfn2 fibroblasts was significantly higher compared with that of the sh-Mfn2-NC and control groups. These results were consistent with a previous study (19); however, in POP patients, increased expression levels of Mfn2 may not be beneficial, as decreased proliferation of fibroblasts may be associated with their dysfunction, which could be the cause of POP.

The present study indicated that the $M f n 2$ gene could cause growth arrest by blocking the cell cycle at the G0/G1 phase (25). Three major classes of proteins are involved in the regulation of the cell cycle: Cyclins, CDK enzymes and cyclin-dependent kinase inhibitors (CDKI) (26). CDK2 is a key kinase that initiates DNA replication required for the $\mathrm{G} 2$ phase transition. CDKI inhibits CDK enzymes that prevent cells from passing through the restriction point. p21 is mainly involved in the regulation of the activity of the CDK proteins (26). The present study indicated that overexpression of Mfn2 in NPOP fibroblasts caused a decrease in the expression levels of CDK2, whereas the expression levels of the p21waf 1 protein were significantly increased, which is associated with G0/G1 phase arrest. Similarly, a higher percentage of cells in the sh-Mfn2 group were able to enter $\mathrm{S}$ phase required for DNA synthesis, and $\mathrm{G} 2 / \mathrm{M}$ phase required for mitotic division, which is characterized by increased CDK2 and decreased p21waf1 levels (25). This conclusion is consistent with our previous results obtained from cell cycle studies $(21,22)$.

The results of the current study indicated that Mfn2 notably inhibited cell proliferation through the ERK signaling pathway (27), which regulates a number of important cellular biological processes, including cell proliferation and differentiation (14). The Ras/Raf/MEK/ERK pathway transmits various downstream signals in order to activate nuclear proteins involved in the aforementioned biological processes (27). Activated ERK can promote the phosphorylation of its cytoplasmic target protein and/or regulate the activity of other protein kinases (14). Our results suggested that the upregulation of Mfn2 in POP fibroblasts inhibited the phosphorylation of these proteins by affecting Ras and Raf-1 proteins, two upstream effectors of ERK1/2, thereby inhibiting cell proliferation.

In conclusion, the present study demonstrated that the Mfn2-overexpression group, the expression levels of the procollagen proteins and cell proliferation were markedly decreased compared with the controls. This indicated that the increase in the levels of Mfn2 in POP fibroblasts inhibited the levels of procollagen proteins, resulting in the decrease of synthesized collagen secreted into the ECM. This could in turn lead to the weakness of the pelvic floor support structure based on the uterosacral ligament. These effects favor the occurrence and development of POP. Furthermore, we also proposed that Mfn2 may affect cell proliferation and the cell cycle via the Ras-Raf-ERK signaling pathway, and CDK2 and p21waf1 proteins. With regards to the causal association between the occurrence of POP and increased Mfn2 expression, Mfn2 was upregulated in the uterosacral ligaments in patients with POP. Our findings may provide insight into the pathogenesis, clinical prediction, individual diagnosis and treatment of POP; however, further investigation is required.

\section{Acknowledgements}

The authors would like to thank Dr Yu Qi from Peking University First Hospital, for his instructive advice and useful 
suggestions on the experiment design. Our abstract entitled 'Mitofusin2 regulates the proliferation and function of fibroblasts: Possible mechanisms of pelvic organ prolapse' (receipt no. 191) was selected as a non-moderated e-poster at the 43rd IUGA Annual Meeting, which took place at the Austria Center, Vienna, Austria on June 27-30, 2018.

\section{Funding}

The present study was supported by a grant from the National Natural Science Foundation of China NSFC (grant no. 81401185$)$.

\section{Availability of data and materials}

The datasets used and/or analyzed during the present study are available from the corresponding author on reasonable request.

\section{Authors' contributions}

YL contibuted to the conception and design of the study, and obtained funding. CP and YZ communicated with patients, collected samples and revised the manuscript. HC was responsible for the acquisition of data. $\mathrm{XqW}$ and $\mathrm{XxQ}$ were responsible for the analysis and interpretation of data. $\mathrm{XqW}$ drafted the manuscript.

\section{Ethics approval and consent to participate}

The samples were obtained following informed consent provided by the patients and the ethics committee of the Peking University First Hospital [2016(1173)] approved the study protocol.

\section{Patient consent for publication}

Not applicable.

\section{Competing interests}

The authors declare that they have no competing interests.

\section{References}

1. Khan AA, Eilber KS, Clemens JQ, Wu N, Pashos CL and Anger JT: Trends in management of pelvic organ prolapse among female Medicare beneficiaries. Am J Obstet Gynecol 212: e1-e8, 2015.

2. Dekker JH: Pelvic organ prolapse: Prevention by training? Lancet 389: 336-337, 2017.

3. Dumoulin C, Hunter KF, Moore K, Bradley CS, Burgio KL, Hagen S, Imamura M, Thakar R, Williams K and Chambers T: Conservative management for female urinary incontinence and pelvic organ prolapse review 2013: Summary of the 5th international consultation on incontinence. Neurourol Urodyn 35: 15-20, 2016.

4. Alves FK, Riccetto C, Adami DB, Marques J, Pereira LC, Palma $\mathrm{P}$ and Botelho S: A pelvic floor muscle training program in postmenopausal women: A randomized controlled trial. Maturitas 81: 300-305, 2015.

5. Bø K, Hilde G, Stær-Jensen J, Siafarikas F, Tennfjord MK and Engh ME: Postpartum pelvic floor muscle training and pelvic organ prolapse-a randomized trial of primiparous women. Am J Obstet Gynecol 212: 38.e1-e7, 2015.
6. Sedgwick P: What is significance? BMJ 350: h3475, 2015.

7. Sung VW, Wohlrab KJ, Madsen A and Raker C: Patient-reported goal attainment and comprehensive functioning outcomes after surgery compared to pessary for pelvic organ prolapse. Am J Obstet Gynecol 215: 659.e1-659.e7, 2016.

8. Jeon MJ, Kim EJ, Lee M, Kim H, Choi JR, Chae HD, Moon YJ, Kim SK and Bai SW: MicroRNA-30d and microRNA-181a regulate HOXA11 expression in the uterosacral ligaments and are overexpressed in pelvic organ prolapse. J Cell Mol Med 19: 501-509, 2015.

9. Ulrich D, Edwards SL, Su K, White JF, Ramshaw JA, Jenkin G, Deprest J, Rosamilia A, Werkmeister JA and Gargett CE: Influence of reproductive status on tissue composition and biomechanical properties of ovine vagina. PLoS One 9: e93172, 2014.

10. Liang R, Zong W, Palcsey S, Abramowitch S and Moalli PA: Impact of prolapse meshes on the metabolism of vaginal extracellular matrix in rhesus macaque. Am J Obstet Gynecol 212: 174.e1-e7, 2015.

11. Kim T, Sridharan I, Ma Y, Zhu B, Chi N, Kobak W, Rotmensch J, Schieber JD and Wang R: Identifying distinct nanoscopic features of native collagen fibrils towards early diagnosis of pelvic organ prolapse. Nanomedicine 12: 667-675, 2016.

12. Liang R, Knight K, Barone W, Powers RW, Nolfi A, Palcsey S, Abramowitch S and Moalli PA: Extracellular matrix regenerative graft attenuates the negative impact of polypropylene prolapse mesh on vagina in rhesus macaque. Am J Obstet Gynecol 216: 153.e1-153.e9, 2017.

13. Wirostko BM, Curtin K, Ritch R, Thomas S, Allen-Brady K, Smith KR, Hageman GS and Allingham RR: Risk for exfoliation syndrome in women with pelvic organ prolapse: A utah project on exfoliation syndrome (UPEXS) study. JAMA Ophthalmol 134: 1255-1262, 2016

14. Tracy LE, Minasian A and Caterson EJ: Extracellular matrix and dermal fibroblast function in the healing wound. Adv Skin Wound Care (New Rochelle) 5: 119-136, 2016.

15. Zorzano A, Hernández-Alvarez MI, Sebastián D and Muñoz JP Mitofusin 2 as a driver that controls energy metabolism and insulin signaling. Antiox Redox Signaling 22: 1020-1031, 2015.

16. Schrepfer E and Scorrano L: Mitofusins from mitochondria to metabolism. Mol Cell 61: 683-694, 2016.

17. Sebastián D and Zorzano A: When MFN2 (mitofusin 2) met autophagy: A new age for old muscles. Autophagy 12: 2250-2251, 2016.

18. Zheng L, Li S, Zhao S and Fa X: Upregulated miR-17 regulates hypoxia-mediated human pulmonary artery smooth muscle cell proliferation and apoptosis by targeting Mitofusin 2. Med Sci Monit 22: 3301-3308, 2016.

19. Lou Y, Li R, Liu J, Zhang Y, Zhang X, Jin B, Liu Y, Wang Z, Zhong $\mathrm{H}$, Wen $\mathrm{S}$ and Han B: Mitofusin-2 over-expresses and leads to dysregulation of cell cycle and cell invasion in lung adenocarcinoma. Med Oncol 32: 132, 2015.

20. Wu JM, Dieter AA, Pate V and Jonsson Funk M: Cumulative incidence of a subsequent surgery after stress urinary incontinence and pelvic organ prolapse procedure. Obstet Gynecol 129: 1124-1130, 2017.

21. Chen HY, Lu Y, Qi Y, Bai WP and Liao QP: Relationship between the expressions of mitofusin-2 and procollagen in uterosacral ligament fibroblasts of postmenopausal patients with pelvic organ prolapse. Eur J Obstet Gynecol Reprod Biol 174: $141-145,2014$.

22. Lu Y, Chen HY, Wang XQ and Wang JX: Correlations between Mitofusin 2 expression in fibroblasts and pelvic organ prolapse: An in vitro study. Chin Med J (Engl) 130: 2951-2959, 2017.

23. Bump RC, Mattiasson A, B $\varnothing$ K, Brubaker LP, DeLancey JO, Klarskov P, Shull BL and Smith AR: The standardization of terminology of female pelvic organ prolapse and pelvic floor dysfunction. Am J Obstet Gynecol 175: 10-17, 1996.

24. Merten OW, Hebben M and Bovolenta C: Production of lentiviral vectors. Mol Ther Methods Clin Dev 3: 16017, 2016.

25. Asghar U, Witkiewicz AK, Turner NC and Knudsen ES: The history and future of targeting cyclin-dependent kinases in cancer therapy. Nat Rev Drug Discov 14: 130-146, 2015.

26. Branzei D and Foiani M: Regulation of DNA repair throughout the cell cycle. Nat Rev Mol Cell Biol 9: 297-308, 2008.

27. Chen KH, Dasgupta A, Ding J, Indig FE, Ghosh P and Longo DL: Role of mitofusin 2 (Mfn2) in controlling cellular proliferation. FASEB J 28: 382-394, 2014. 